\title{
Acid-catalyzed intramolecular oxa-Michael addition reactions under solvent-free and microwave irradiation conditions
}

\author{
Yi-Ming Hong, Zhen-Lu Shen,* Xin-Quan Hu, Wei-Min Mo, Xiao-Fei He, Bao-Xiang Hu, \\ and Nan Sun
}

College of Chemical Engineering and Materials Science, State Key Laboratory Breeding Base of Green Chemistry-Synthesis Technology, Zhejiang University of Technology, Hangzhou, Zhejiang 310014, China

E-mail: zhenlushen@,zjut.edu.cn

\begin{abstract}
The acid-catalyzed intramolecular oxa-Michael addition of (E)-1-aryl-4-hydroxy-4-methyl-pent-1-en-3-ones under solvent-free and microwave irradiation conditions has been investigated. The results showed that Bronsted acids are more efficient than Lewis acids in this reaction. Up to $90 \%$ conversion and $81 \%$ yield were obtained using trifluoromethanesulfonic acid (triflic acid) as the catalyst, with short reaction times and an environmentally benign procedure.
\end{abstract}

Keywords: Microwave, oxa-Michael addition, solvent-free, trifluoromethanesulfonic acid, (E)-1-aryl-4-hydroxy-4-methylpent-1-en-3-ones

\section{Introduction}

Organic chemists are constantly challenged to develop environmentally benign routes for preparing desired target compounds. Microwave irradiation has emerged as a convenient and powerful method for promoting organic reactions, owing to its uniform heating effect. ${ }^{1-3}$ Another green chemistry principle - solvent-free organic synthesis - has also received much attention from organic chemists. The combination of solvent-free and microwave irradiation can lead to large reductions in reaction times, or enhancements in conversions, and will make synthesis more environmentally benign. ${ }^{4-6}$

Michael and hetero-Michael additions are effective for the formation of carbon-carbon and carbon- hetero-atom bonds, and have been utilized widely in inter- and intra- molecular reactions to obtain various products, which include important building blocks for many biologically active molecules. ${ }^{7,8}$ Recently, the conjugate addition of oxygen nucleophiles to $\alpha, \beta$-unsaturated carbonyl compounds (the oxa-Michael addition) has attracted much attention. ${ }^{9}$ Various catalysts have been reported for intermolecular oxa-Michael additions, such as pro-azaphosphatranes 
$\left(\mathrm{P}\left(\mathrm{RNCH}_{2} \mathrm{CH}_{2}\right)_{3} \mathrm{~N}\right),{ }^{10}$ trimethylphosphine, tributylphosphine, ${ }^{11}$ 1,8-diazabicyclo[5.4.0]undec-7ene (DBU), ${ }^{12}[\mathrm{Rh}(\mathrm{COD})(\mathrm{OMe})]_{2},{ }^{13}\left(\mathrm{CH}_{3} \mathrm{CN}\right)_{2} \mathrm{PdCl}_{2},{ }^{14}$ (1,3-bis-(2,6-di-isopropylphenyl)imidazol2-yliden $) \mathrm{Cu}(\mathrm{OEt}), \quad$ (1,3-bis-(2,6-di-isopropylphenyl)imidazol-2-yliden) $\mathrm{Cu}(\mathrm{OPh}), \quad$ (1,3-bis-(2,6di-isopropylphenyl)imidazolin-2-ylidene) $\mathrm{Cu}(\mathrm{OEt}),{ }^{15} \mathrm{KF} / \mathrm{Al}_{2} \mathrm{O}_{3},{ }^{16}\left(\mathrm{CF}_{3} \mathrm{SO}_{2}\right)_{2} \mathrm{NH},{ }^{17} \mathrm{BF}_{3} \cdot \mathrm{Et}_{2} \mathrm{O},{ }^{18}$ $\mathrm{La}\left(\mathrm{NO}_{3}\right)_{3} \cdot 6 \mathrm{H}_{2} \mathrm{O},{ }^{19}$ pyrrolidine $/ \mathrm{CH}_{3} \mathrm{SO}_{3} \mathrm{H}^{20}$ and biphenyldiamine-based organocatalyst. ${ }^{21}$ The use of ionic liquid as solvent for toluenesulfonic acid-catalyzed oxa-Michael addition has also been reported. ${ }^{22}$ For intramolecular oxa-Michael addition, cinchona alkaloids, ${ }^{23}$ thiourea derivatives, ${ }^{24}$ quinine, ${ }^{25}$ guanidine, ${ }^{26}\left[\mathrm{Pd}\left(\mathrm{MeCN}_{4}\right)\right]\left(\mathrm{BF}_{4}\right)_{2},{ }^{27} \mathrm{~N}, \mathrm{~N}$-dioxide nickel(II) complex, ${ }^{28} \mathrm{CF}_{3} \mathrm{COOH}^{29}$ and toluenesulfonic acid $(\mathrm{TsOH})^{30,31}$ have been utilized as the catalysts. It was reported that the intramolecular oxa-Michael addition of (E)-1-aryl-4-hydroxy-4-methylpent-1-en-3-ones in the presence of $\mathrm{TsOH}$ should be carried out by refluxing in dichloroethane for $24 \mathrm{~h}$, while the products were hard to purify due to the impurities. ${ }^{31}$

In continuation of our work on "green" chemistry, ${ }^{32}$ we report herein an efficient acid-catalyzed intramolecular oxa-Michael addition reaction of (E)-1-aryl-4-hydroxy-4-methylpent-1-en-3-ones 1 under solvent-free and microwave irradiation conditions.

\section{Results and Discussion}

Effect of catalysts. In order to find the most efficient catalyst, we studied first the intramolecular oxa-Michael addition of (E)-4-hydroxy-4-methyl-1-phenylpent-1-en-3-one 1a under solvent-free and microwave irradiation conditions. The results are given in Table 1.

Table 1. Effect of catalysts in the intramolecular oxa-Michael addition of $\mathbf{1} \mathbf{a}^{\mathrm{a}}$

\begin{tabular}{cccccc}
\hline Entry & Catalyst & $T_{\text {final }}\left({ }^{\circ} \mathrm{C}\right)$ & Conversion $(\%)^{\mathrm{b}}$ & ${\text { Selectivity }(\%)^{\mathrm{b}}}$ & ${\text { Yield }(\%)^{\mathrm{c}}}^{\mathrm{c}}$ \\
\hline 1 & $\mathrm{Zn}(\mathrm{OTf})_{2}$ & 141 & 32 & 83 & 20 \\
2 & $\mathrm{Cu}(\mathrm{OTf})_{2}$ & 143 & 59 & 87 & 46 \\
3 & $\mathrm{TsOH} \cdot \mathrm{H}_{2} \mathrm{O}$ & 118 & 69 & 91 & 57 \\
4 & $\mathrm{CH}_{3} \mathrm{SO}_{3} \mathrm{H}$ & 115 & 64 & 97 & 58 \\
5 & $\mathrm{TfOH}$ & 117 & 88 & 94 & 78 \\
6 & $\mathrm{DBU}$ & 116 & 0 & - & - \\
7 & $\mathrm{DABCO}$ & 116 & 0 & - & - \\
8 & $\left(\mathrm{CH}_{3} \mathrm{CN}\right)_{2} \mathrm{PdCl}_{2}$ & 165 & 96 & 16 & - \\
\hline
\end{tabular}

${ }^{a}$ Reaction conditions: $1 \mathrm{a}(0.5 \mathrm{mmol})$, catalyst $(0.05 \mathrm{mmol}), 650 \mathrm{~W}$ microwave power, $9 \mathrm{~min}$ total reaction time, with alternation between $9 \mathrm{~s}$ of irradiating time and $21 \mathrm{~s}$ of cooling time.

b Conversion of 1a and selectivity to $\mathbf{2 a}$ were measured on the reaction mixture by gas chromatography. ${ }^{\mathrm{c}}$ Isolated yield. 
When the Lewis acid zinc trifluoromethanesulfonate $\left(\mathrm{Zn}(\mathrm{OTf})_{2}\right)$ was used as catalyst, the conversion of $\mathbf{1 a}$ and the selectivity to 2,2-dimethyl-5-phenyl-dihydrofuran-3(2H)-one $\mathbf{2 a}$ were 32 and $83 \%$, respectively, and the isolated yield of $\mathbf{2 a}$ was only $20 \%$ (entry 1 ). The other Lewis acid, copper trifluoromethanesulfonate $\left(\mathrm{Cu}(\mathrm{OTf})_{2}\right)$ showed higher activity, and a $59 \%$ conversion with $87 \%$ selectivity was obtained (entry 2). However, it was found that Bronsted acids are more efficient than Lewis acids in this reaction (entries 3-5). Among the tested Bronsted acids trifluoromethanesulfonic acid (triflic acid, TfOH), TsOH, and $\mathrm{CH}_{3} \mathrm{SO}_{3} \mathrm{H}$, the $\mathrm{TfOH}$ gave the best result, with up to $88 \%$ conversion and $94 \%$ selectivity (entry 5). Organic bases, such as DBU and 1,4-diazabicyclo[2.2.2] octane (DABCO), were also examined but showed no activity in this intramolecular oxa-Michael addition (entries 6 and 7), although they have been employed as catalysts in some intermolecular oxa-Michael additions. ${ }^{12,33}$ The highest conversion $(96 \%)$ of $1 \mathbf{a}$ was observed in the presence of $\left(\mathrm{CH}_{3} \mathrm{CN}\right)_{2} \mathrm{PdCl}_{2}$, but many unidentified by-products were observed and the selectivity to 2 a was only $16 \%$ (entry 8 ).

\section{Comparison of plausible reaction mechanism in the presence of Bronsted acids and Lewis acid}

A plausible reaction mechanism for the intramolecular oxa-Michael addition of 1a, promoted with Bronsted acid, as given by Johnson's group, is outlined in Scheme 1. 31,34 The carbonyl oxygen of 1a accepts one proton from the Bronsted acid to give the protonated form I and canonical form II, and undergoes ring closure to give the enol form III of $\mathbf{2 a}$.

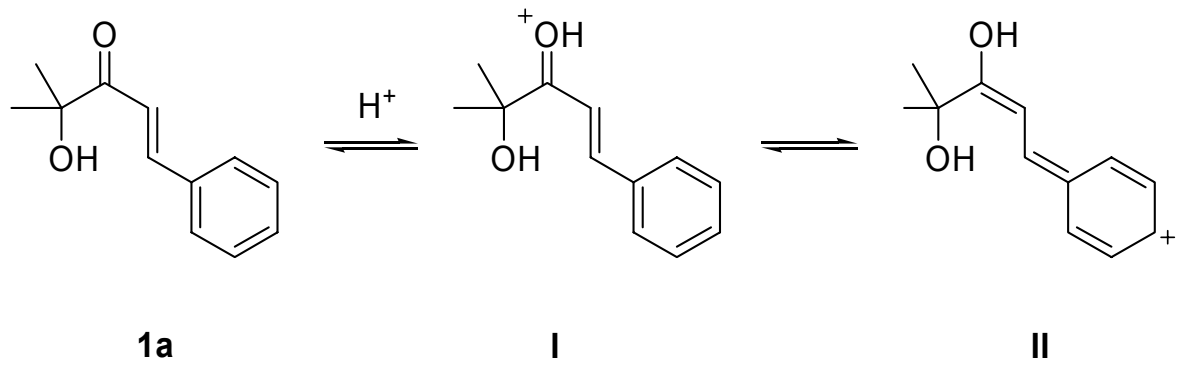

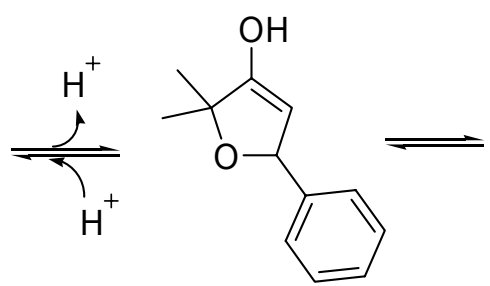

III

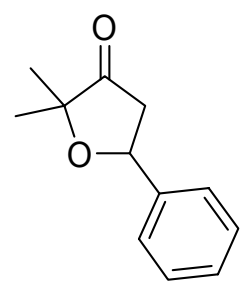

$2 a$

Scheme 1. Plausible reaction mechanism in the presence of Bronsted acids. 
It is presumed that in the presence of Bronsted acid, there exists an equilibrium between 1a and $2 \mathbf{a} .{ }^{31}$ When a mixture of $2 \mathrm{a}(0.5 \mathrm{mmol})$ and TfOH $(0.05 \mathrm{mmol})$ was irradiated at $650 \mathrm{~W}$ for 9 min, it was found that $13 \%$ of $\mathbf{2 a}$ did convert into $\mathbf{1 a}$.

Spencer and colleagues have reported that protons generated through in situ hydrolysis of metal salts were the active catalysts in some Lewis acid mediated hetero-Michael additions. ${ }^{35}$ In order to ascertain whether protons are the actual catalysts in this intramolecular oxa-Michael addition in the presence of Lewis acids, $\mathrm{Zn}(\mathrm{OTf})_{2}$ and $\mathrm{Cu}(\mathrm{OTf})_{2}$, the above experiment was repeated using $\mathrm{Zn}(\mathrm{OTf})_{2}$ in place of $\mathrm{TfOH}$. The experiments showed that no $\mathbf{2 a}$ was converted into 1a after irradiating for $9 \mathrm{~min}$, while our previous experiments showed both Lewis acids and Bronsted acids could catalyze this intramolecular oxa-Michael reaction (Table 1, entries 1 and 2 versus 3-5). These results suggest that protons were not the active catalysts in this Lewis acid-catalyzed oxa-Michael addition. Based on our experimental results we propose a plausible reaction mechanism shown in Scheme $2 . \mathrm{Zn}(\mathrm{OTf})_{2}$ and $\mathrm{Cu}(\mathrm{OTf})_{2}$ are highly oxophilic, and could form a carbonyl- metal-ion complex IV with 1a, which would initiate the formation of a C-O bond to generate the intermediate $\mathbf{V}$. This could regenerate the catalyst and give the intermediate VI. After the intramolecular proton transfer step, 2a was obtained.
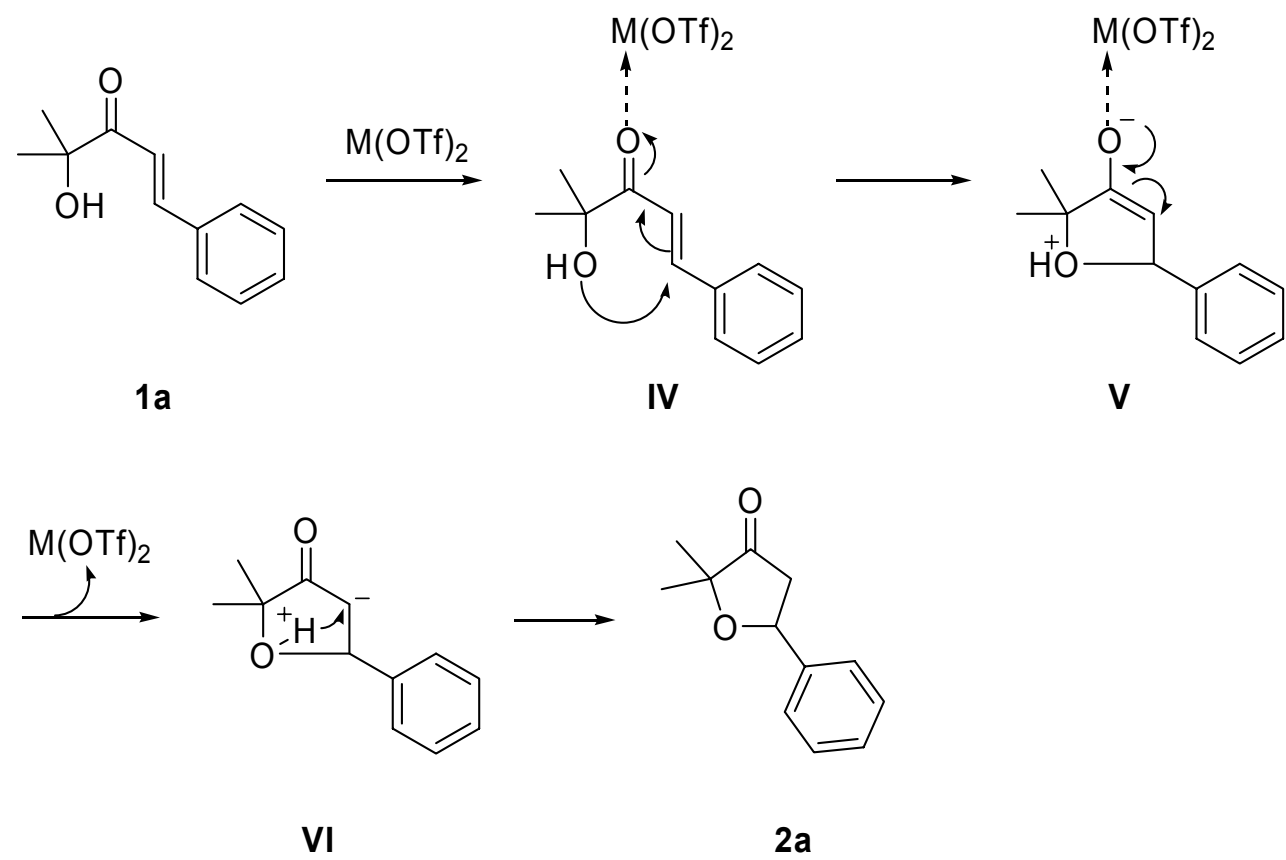

Scheme 2. Plausible reaction mechanism in the presence of $\mathrm{M}(\mathrm{OTf})_{2}$.

\section{Intramolecular oxa-Michael addition of (E)-1-aryl-4-hydroxy-4-methylpent-1-en-3-ones}

To evaluate the scope of the intramolecular oxa-Michael addition, substrates with different steric property and electron property have been investigated using $\mathrm{TfOH}$ as the catalyst under the microwave-assisted process. As shown in Table 2, most of the reactions proceeded efficiently, high conversions of (E)-1-aryl-4-hydroxy-4-methylpent-1-en-3-ones 1 and selectivities to the 
desired products 5-aryl-2,2-dimethyl-dihydrofuran-3(2H)-ones 2 were obtained within several minutes.

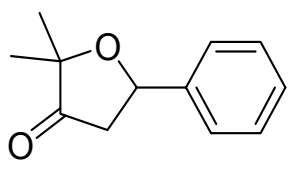

2a

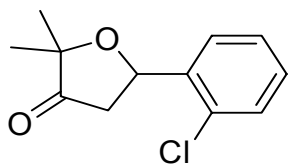

2e

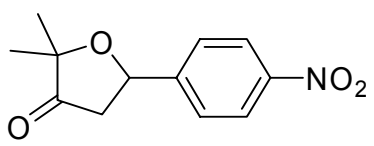

$2 \mathbf{i}$

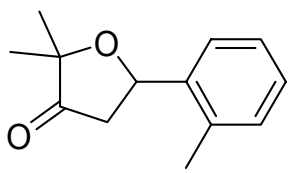

2b

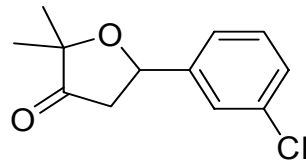

2f

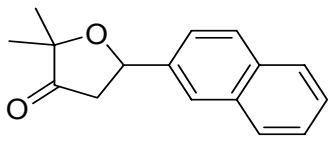

$\mathbf{2 j}$

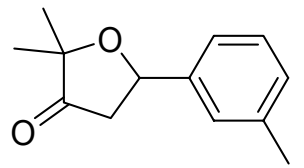

2c

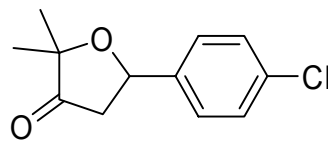

$2 \mathbf{g}$

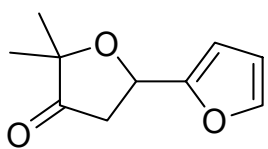

$2 \mathbf{k}$

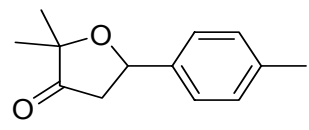

2d

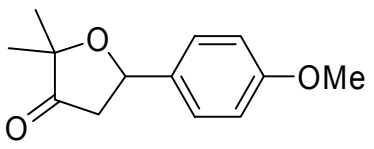

$2 h$

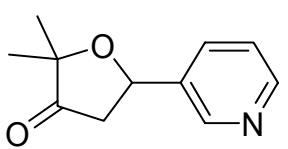

21

It was observed that the position of substituents on the benzene ring had little effect on the conversions and selectivities (entries 2-4, methyl as substituent; entries 5-6, chlorine as substituent). Also, the electronic properties of substituents on the benzene ring showed marginal effects on the conversions and selectivities (entry 4, $p$-Me; entry $7, p$ - $\mathrm{Cl}$; entry $8, p$ - $\mathrm{OCH}_{3}$ ), even with a strongly electron-withdrawing group (entry 9, $p-\mathrm{NO}_{2}$ ). A similar result was obtained when a naphthalene ring replaced a benzene ring, the conversion of $\mathbf{1} \mathbf{j}$ and selectivity to $\mathbf{2} \mathbf{j}$ were 84 and 95\%, respectively (entry 10 ). When (E)-1-(furan-2-yl)-4-hydroxy-4-methylpent-1-en-3-one 1k was used as the substrate, the irradiating period and reaction time must be shortened. Otherwise, the selectivity to $\mathbf{2 k}$ would drastically decrease, which may be due to the polymerization of $\mathbf{2 k}$. Under our conditions, $17 \%$ conversion of $\mathbf{1} \mathbf{k}$ and $8 \%$ isolated yield of $\mathbf{2} \mathbf{k}$ were obtained (entry 11 ). The intramolecular oxa-Michael addition of (E)-4-hydroxy-4-methyl-1-(pyridin-3-yl)-pent-1-en-3-one 11 could not occur under microwave irradiation or refluxing conditions because the proton of Bronsted acid would be captured by the $\mathrm{N}$ - atom of the pyridinyl group. 
Table 2. Intramolecular oxa-Michael addition of (E)-1-aryl-4-hydroxy-4-methylpent-1-en-3-ones under solvent-free and microwave conditions ${ }^{\mathrm{a}}$

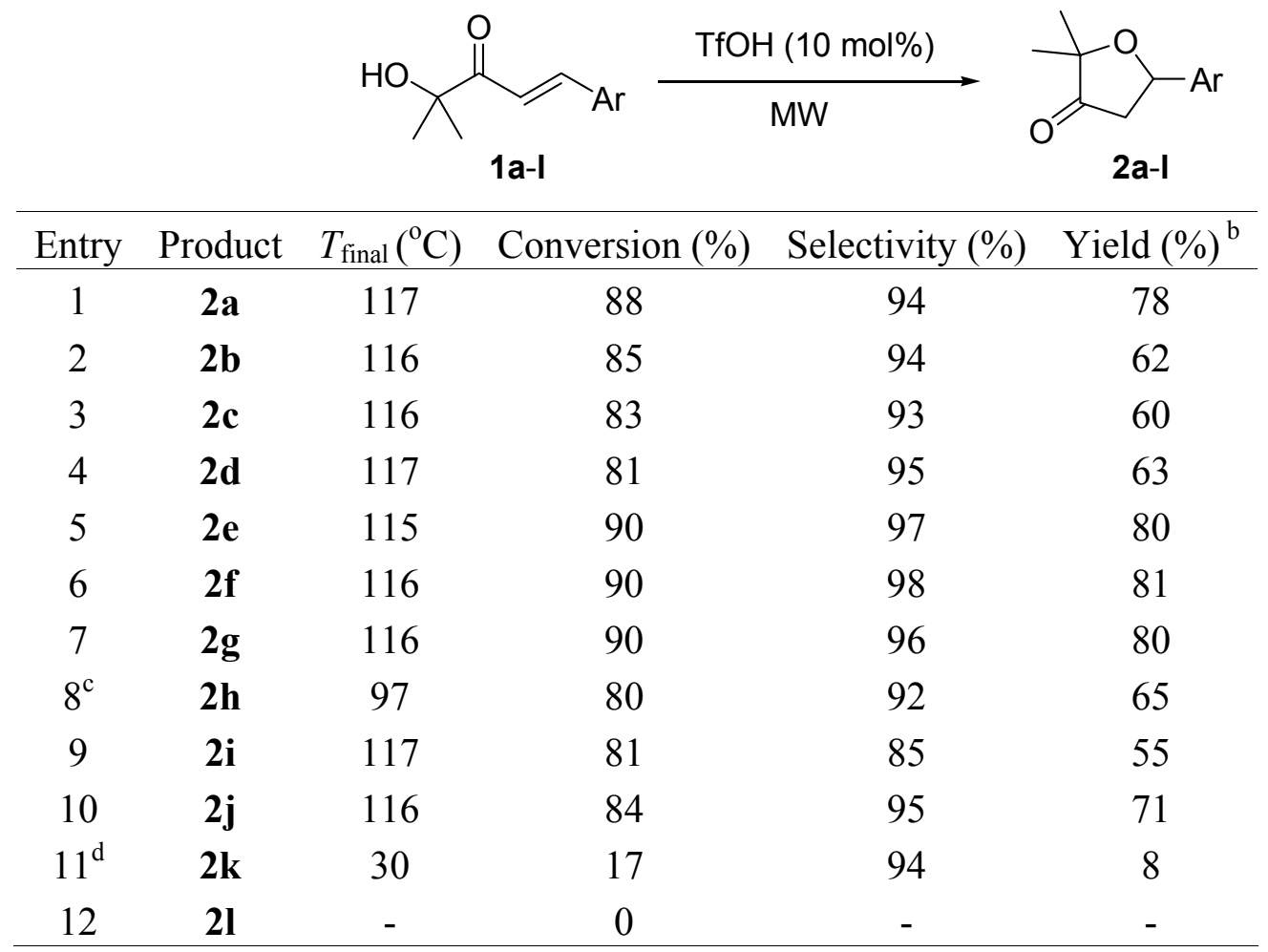

${ }^{a}$ Reaction conditions: 1 ( $\left.0.5 \mathrm{mmol}\right)$, TfOH $(0.05 \mathrm{mmol}), 650 \mathrm{~W}$ (microwave power), 9 min (total reaction time, with an alternation between $9 \mathrm{~s}$ of irradiating time and $21 \mathrm{~s}$ of cooling time).

${ }^{\mathrm{b}}$ Isolated yield. ${ }^{\mathrm{c}}$ With an alternation between $6 \mathrm{~s}$ of irradiating time and $24 \mathrm{~s}$ of cooling time. ${ }^{\mathrm{d}}$ Total reaction time $3 \mathrm{~min}$, with an alternation between $3 \mathrm{~s}$ of irradiating time and $27 \mathrm{~s}$ of cooling time.

\section{Conclusions}

The acid-catalyzed intramolecular oxa-Michael addition of (E)-1-aryl-4-hydroxy-4-methylpent-1-en-3-ones could be performed under solvent-free and microwave irradiation conditions, providing an environmentally benign method for preparing 5-aryl-2,2-dimethyl-dihydrofuran$3(2 \mathrm{H})$-ones. It was found that Bronsted acids are more efficient than Lewis acids in this reaction. Unlike traditional reactions, the solvent-free and microwave-assisted reactions could be finished within several minutes to give high conversions and selectivities. 


\section{Experimental Section}

General. ${ }^{1} \mathrm{H}$ - and ${ }^{13} \mathrm{C}$ - NMR spectra were obtained on a Bruker Avance III (500 MHz) spectrometer. $\mathrm{CDCl}_{3}$ was used as the solvent with tetramethylsilane (TMS) as the internal standard. Low and high resolution mass spectra were recorded in the EI mode on a Waters GCT Premier mass spectrometer. Melting points were measured using CRC-1 melting point instrument and are uncorrected. Microwave experiments were performed at a LWMC-201 microwave reactor. The reaction temperature was determined by an IR thermometer (SUN-GUN SG-20). Reactions were monitored by gas chromatography (GC-6890) with an HP-5 capillary column (30m $\mathrm{x}$ $0.25 \mathrm{~mm})$. All reagents were obtained from commercial sources and used as received. (E)-1-Aryl-4-hydroxy-4-methylpent-1-en-3-ones were prepared as previously described. ${ }^{30,36}$

General procedure for the synthesis of 5-aryl-2,2-dimethyl-dihydrofuran-3(2H)-ones (2a-k) To an open glass tube, were added (E)-1-aryl-4-hydroxy-4-methylpent-1-en-3-one (0.5 mmol) and triflic acid $(0.05 \mathrm{mmol})$. The tube was positioned in the centre of the microwave cavity, and irradiated $(650 \mathrm{~W})$ for $9 \mathrm{~min}$ (total reaction time, with alternation of $9 \mathrm{~s}$ irradiating time and $21 \mathrm{~s}$ of cooling time). After the last period of irradiating, the temperature (on the surface of the tube) was determined by an IR thermometer. After cooling, the reaction mixture was diluted with $\mathrm{CH}_{2} \mathrm{Cl}_{2}$ and purified through a column of silica gel to obtain the pure products $\mathbf{2 a - k}$.

2,2-Dimethyl-5-phenyl-dihydrofuran-3(2H)-one (2a). Yellow oil. ${ }^{1} \mathrm{H}$ NMR $\left(\mathrm{CDCl}_{3}\right) \delta 1.33$ (s, $\left.3 \mathrm{H},-\mathrm{CH}_{3}\right), 1.41\left(\mathrm{~s}, 3 \mathrm{H},-\mathrm{CH}_{3}\right), 2.56(\mathrm{dd}, J=10.5 \mathrm{~Hz} ; J=18 \mathrm{~Hz}, 1 \mathrm{H}, \mathrm{H}-4 \mathrm{a}), 2.87$ (dd, $J=6 \mathrm{~Hz} ; J=$ $18.5 \mathrm{~Hz}, 1 \mathrm{H}, \mathrm{H}-4 \mathrm{~b}), 5.21$ (dd, $J=10.5 \mathrm{~Hz} ; J=6 \mathrm{~Hz}, 1 \mathrm{H},-\mathrm{CH}), 7.30-7.34$ (m, 1H, phenyl-H), 7.37-7.42 (m, 4H, phenyl-H). ${ }^{13} \mathrm{C}$ NMR $\left(\mathrm{CDCl}_{3}\right) \delta 21.70\left(-\mathrm{CH}_{3}\right), 24.47\left(-\mathrm{CH}_{3}\right), 44.24(\mathrm{C}-4), 74.51$ (C-5), 81.51 (C-2), 126.13 (phenyl-C), 128.32 (phenyl-C), 128.78 (phenyl-C), 140.69 (phenyl-C), $217.35(\mathrm{O}=\mathrm{C})$. MS (EI), $m / z(\%) 190.1\left(\mathrm{M}^{+}, 2\right), 104.1(100)$.

2,2-Dimethyl-5-o-tolyl-dihydrofuran-3(2H)-one (2b). Yellow oil. ${ }^{1} \mathrm{H}$ NMR $\left(\mathrm{CDCl}_{3}\right) \delta 1.35$ (s, $\left.3 \mathrm{H},-\mathrm{CH}_{3}\right), 1.43\left(\mathrm{~s}, 3 \mathrm{H},-\mathrm{CH}_{3}\right), 2.34\left(\mathrm{~s}, 3 \mathrm{H}\right.$, phenyl- $\left.\mathrm{CH}_{3}\right), 2.46(\mathrm{dd}, J=10.5 \mathrm{~Hz} ; J=18 \mathrm{~Hz}, 1 \mathrm{H}$, H-4a), 2.90 (dd, $J=6 \mathrm{~Hz} ; J=18 \mathrm{~Hz}, 1 \mathrm{H}, \mathrm{H}-4 \mathrm{~b}), 5.38$ (dd, $J=10.5 \mathrm{~Hz} ; J=6 \mathrm{~Hz}, 1 \mathrm{H},-\mathrm{CH}), 7.17$ (d, $J=7.5 \mathrm{~Hz}, 1 \mathrm{H}$, phenyl-H), 7.20-7.26 (m, 2H, phenyl-H), $7.56\left(\mathrm{~d}, 1 \mathrm{H}\right.$, phenyl-H). ${ }^{13} \mathrm{C}$ NMR $\left(\mathrm{CDCl}_{3}\right) \delta 19.19$ (phenyl-CH3), $21.60\left(-\mathrm{CH}_{3}\right), 24.31\left(-\mathrm{CH}_{3}\right), 42.82(\mathrm{C}-4), 71.44(\mathrm{C}-5), 81.11(\mathrm{C}-2)$, 124.77 (phenyl-C), 126.52 (phenyl-C), 127.80 (phenyl-C), 130.41 (phenyl-C), 134.80 (phenyl-C), 138.79 (phenyl-C), $217.46(\mathrm{O}=\mathrm{C})$. MS (EI), $m / z(\%) 204.1\left(\mathrm{M}^{+}, 3\right), 118.1(100)$. HRMS Calcd. for $\mathrm{C}_{13} \mathrm{H}_{16} \mathrm{O}_{2} 204.1150$, found 204.1158.

2,2-Dimethyl-5-m-tolyl-dihydrofuran-3(2H)-one (2c). Yellow oil. ${ }^{1} \mathrm{H}$ NMR $\left(\mathrm{CDCl}_{3}\right) \delta 1.32$ (s, $\left.3 \mathrm{H},-\mathrm{CH}_{3}\right), 1.41\left(\mathrm{~s}, 3 \mathrm{H},-\mathrm{CH}_{3}\right), 2.37\left(\mathrm{~s}, 3 \mathrm{H}\right.$, phenyl- $\left.\mathrm{CH}_{3}\right), 2.57$ (dd, $J=10.5 \mathrm{~Hz} ; J=18 \mathrm{~Hz}, 1 \mathrm{H}$, H-4a), 2.86 (dd, $J=6 \mathrm{~Hz} ; J=18 \mathrm{~Hz}, 1 \mathrm{H}, \mathrm{H}-4 \mathrm{~b}$ ), 5.18 (dd, $J=10.5 \mathrm{~Hz} ; J=6 \mathrm{~Hz}, 1 \mathrm{H},-\mathrm{CH}), 7.14$ (d, $J=7.5 \mathrm{~Hz}, 1 \mathrm{H}$, phenyl-H), 7.20 (d, $J=8 \mathrm{~Hz}, 1 \mathrm{H}$, phenyl-H), $7.23(\mathrm{~s}, 1 \mathrm{H}$, phenyl-H), 7.27 (t, $J=8$ $\mathrm{Hz}, 1 \mathrm{H}$, phenyl-H). ${ }^{13} \mathrm{C} \mathrm{NMR}\left(\mathrm{CDCl}_{3}\right) \delta 21.47$ (phenyl- $\left.\mathrm{CH}_{3}\right), 21.60\left(-\mathrm{CH}_{3}\right), 24.39\left(-\mathrm{CH}_{3}\right), 44.13$ (C-4), 74.47 (C-5), 81.43 (C-2), 123.16 (phenyl-C), 126.66 (phenyl-C), 128.58 (phenyl-C), 129.00 
(phenyl-C), 138.44 (phenyl-C), 140.44 (phenyl-C), $217.42(\mathrm{O}=\mathrm{C}) . \mathrm{MS}(\mathrm{EI}), \mathrm{m} / z(\%) 204.1\left(\mathrm{M}^{+}\right.$, 3), 118.1 (100).

2,2-Dimethyl-5-p-tolyl-dihydrofuran-3(2H)-one (2d). Yellow oil. ${ }^{1} \mathrm{H}$ NMR $\left(\mathrm{CDCl}_{3}\right) \delta 1.32$ (s, $\left.3 \mathrm{H},-\mathrm{CH}_{3}\right), 1.39\left(\mathrm{~s}, 3 \mathrm{H},-\mathrm{CH}_{3}\right), 2.36\left(\mathrm{~s}, 3 \mathrm{H}\right.$, phenyl- $\left.\mathrm{CH}_{3}\right), 2.56(\mathrm{dd}, J=10.5 \mathrm{~Hz} ; J=18 \mathrm{~Hz}, 1 \mathrm{H}$, $\mathrm{H}-4 \mathrm{a}), 2.85$ (dd, $J=6 \mathrm{~Hz} ; J=18.5 \mathrm{~Hz}, 1 \mathrm{H}, \mathrm{H}-4 \mathrm{~b}), 5.18$ (dd, $J=10.5 \mathrm{~Hz} ; J=6 \mathrm{~Hz}, 1 \mathrm{H},-\mathrm{CH}), 7.19$ (d, $J=8 \mathrm{~Hz}, 2 \mathrm{H}$, phenyl-H), 7.30 (d, $J=8 \mathrm{~Hz}, 2 \mathrm{H}$, phenyl-H). ${ }^{13} \mathrm{C} \mathrm{NMR}\left(\mathrm{CDCl}_{3}\right) \delta 21.18$ (phenyl- $\left.\mathrm{CH}_{3}\right), 21.62\left(-\mathrm{CH}_{3}\right), 24.41\left(-\mathrm{CH}_{3}\right), 44.15$ (C-4), 74.33 (C-5), 81.39 (C-2), 126.03 (phenyl-C), 129.34 (phenyl-C), 137.54 (phenyl-C), 138.02 (phenyl-C), $217.54(\mathrm{O}=\mathrm{C})$. MS (EI), $m / z(\%) 204.1\left(\mathrm{M}^{+}, 3\right), 118.1(100)$.

5-(2-Chlorophenyl)-2,2-dimethyl-dihydrofuran-3(2H)-one (2e). Yellow oil. ${ }^{1} \mathrm{H} \mathrm{NMR}\left(\mathrm{CDCl}_{3}\right)$ $\delta 1.35\left(\mathrm{~s}, 3 \mathrm{H},-\mathrm{CH}_{3}\right), 1.44\left(\mathrm{~s}, 3 \mathrm{H},-\mathrm{CH}_{3}\right), 2.33(\mathrm{dd}, J=10.5 \mathrm{~Hz} ; J=18 \mathrm{~Hz}, 1 \mathrm{H}, \mathrm{H}-4 \mathrm{a}), 3.13$ (dd, $J=$ $6 \mathrm{~Hz} ; J=18.5 \mathrm{~Hz}, 1 \mathrm{H}, \mathrm{H}-4 \mathrm{~b}), 5.19$ (dd, $J=10.5 \mathrm{~Hz} ; J=6 \mathrm{~Hz}, 1 \mathrm{H},-\mathrm{CH}), 7.23-7.27$ (m, 3H, phenyl-H), 7.32-7.37 (m, 1H, phenyl-H), 7.67-7.67 (m, 1H, phenyl-H). ${ }^{13} \mathrm{C} \mathrm{NMR}\left(\mathrm{CDCl}_{3}\right) \delta 21.71$ $\left(-\mathrm{CH}_{3}\right), 24.21\left(-\mathrm{CH}_{3}\right), 42.67(\mathrm{C}-4), 71.35$ (C-5), 81.27 (C-2), 126.37 (phenyl-C), 127.33 (phenyl-C), 128.91 (phenyl-C), 129.36 (phenyl-C), 131.90 (phenyl-C), 138.97 (phenyl-C), 216.74 $(\mathrm{O}=\mathrm{C})$. MS (EI), $m / z(\%) 223.9\left(\mathrm{M}^{+}, 2\right), 137.6(100)$. HRMS Calcd. for $\mathrm{C}_{12} \mathrm{H}_{13} \mathrm{O}_{2} \mathrm{Cl} 224.0604$, found 224.0610 .

5-(3-Chlorophenyl)-2,2-dimethyl-dihydrofuran-3(2H)-one (2f). Yellow oil. ${ }^{1} \mathrm{H} \mathrm{NMR}\left(\mathrm{CDCl}_{3}\right)$ $\delta 1.32\left(\mathrm{~s}, 3 \mathrm{H},-\mathrm{CH}_{3}\right), 1.41\left(\mathrm{~s}, 3 \mathrm{H},-\mathrm{CH}_{3}\right), 2.50(\mathrm{dd}, J=10.5 \mathrm{~Hz} ; J=18 \mathrm{~Hz}, 1 \mathrm{H}, \mathrm{H}-4 \mathrm{a}), 2.80$ (dd, $J=$ $6 \mathrm{~Hz} ; J=18 \mathrm{~Hz}, 1 \mathrm{H}, \mathrm{H}-4 \mathrm{~b}), 5.19$ (dd, $J=10.5 \mathrm{~Hz} ; J=6 \mathrm{~Hz}, 1 \mathrm{H},-\mathrm{CH}), 7.26-7.33$ (m, 3H, phenyl-H), $7.42\left(\mathrm{~s}, 1 \mathrm{H}\right.$, phenyl-H). ${ }^{13} \mathrm{C} \mathrm{NMR}\left(\mathrm{CDCl}_{3}\right) \delta 21.59\left(-\mathrm{CH}_{3}\right), 24.32\left(-\mathrm{CH}_{3}\right), 44.00(\mathrm{C}-4)$, 73.71 (C-5), 81.59 (C-2), 124.12 (phenyl-C), 126.09 (phenyl-C), 128.31 (phenyl-C), 129.98 (phenyl-C), 134.64 (phenyl-C), 142.79 (phenyl-C), 216.53 (O=C). MS (EI), m/z (\%) $224.1\left(\mathrm{M}^{+}\right.$, 2), 138.0 (100).

5-(4-Chlorophenyl)-2,2-dimethyl-dihydrofuran-3(2H)-one (2g). Yellow oil. ${ }^{1} \mathrm{H} \mathrm{NMR}\left(\mathrm{CDCl}_{3}\right)$ $\delta 1.32\left(\mathrm{~s}, 3 \mathrm{H},-\mathrm{CH}_{3}\right), 1.40\left(\mathrm{~s}, 3 \mathrm{H},-\mathrm{CH}_{3}\right), 2.49$ (dd, $\left.J=10 \mathrm{~Hz} ; J=18 \mathrm{~Hz}, 1 \mathrm{H}, \mathrm{H}-4 \mathrm{a}\right), 2.87$ (dd, $J=6$ $\mathrm{Hz} ; J=18 \mathrm{~Hz}, 1 \mathrm{H}, \mathrm{H}-4 \mathrm{~b}), 5.19$ (dd, $J=10 \mathrm{~Hz} ; J=6 \mathrm{~Hz}, 1 \mathrm{H},-\mathrm{CH}), 7.33-7.37$ (m, 4H, phenyl-H). ${ }^{13} \mathrm{C} \mathrm{NMR}\left(\mathrm{CDCl}_{3}\right) \delta 21.71\left(-\mathrm{CH}_{3}\right), 24.46\left(-\mathrm{CH}_{3}\right), 44.19(\mathrm{C}-4), 73.86(\mathrm{C}-5), 81.67(\mathrm{C}-2), 127.49$ (phenyl-C), 128.97 (phenyl-C), 134.02 (phenyl-C), 139.33 (phenyl-C), $216.84(\mathrm{O}=\mathrm{C})$. MS (EI), $m / z(\%) 224.1\left(\mathrm{M}^{+}, 2\right), 138.0(100)$.

5-(4-Methoxyphenyl)-2,2-dimethyl-dihydrofuran-3(2H)-one (2h). Yellow oil. ${ }^{1} \mathrm{H}$ NMR $\left(\mathrm{CDCl}_{3}\right) \delta 1.32\left(\mathrm{~s}, 3 \mathrm{H},-\mathrm{CH}_{3}\right), 1.39\left(\mathrm{~s}, 3 \mathrm{H},-\mathrm{CH}_{3}\right), 2.56(\mathrm{dd}, J=10.5 \mathrm{~Hz} ; J=18 \mathrm{~Hz}, 1 \mathrm{H}, \mathrm{H}-4 \mathrm{a}), 2.83$ $(\mathrm{dd}, J=6 \mathrm{~Hz} ; J=18 \mathrm{~Hz}, 1 \mathrm{H}, \mathrm{H}-4 \mathrm{~b}), 3.81\left(\mathrm{~s}, 3 \mathrm{H},-\mathrm{OCH}_{3}\right), 5.16(\mathrm{dd}, J=10.5 \mathrm{~Hz} ; J=6 \mathrm{~Hz}, 1 \mathrm{H}$, -CH), 6.90-6.93 (m, 2H, phenyl-H), 7.33-7.35 (m, 2H, phenyl-H). ${ }^{13} \mathrm{C} \mathrm{NMR}\left(\mathrm{CDCl}_{3}\right) \delta 21.59$ $\left(-\mathrm{CH}_{3}\right), 24.42\left(-\mathrm{CH}_{3}\right), 44.13(\mathrm{C}-4), 55.34\left(-\mathrm{OCH}_{3}\right), 74.17$ (C-5), 81.39 (C-2), 114.06 (phenyl-C), 127.47 (phenyl-C), 132.49 (phenyl-C), 159.58 (phenyl-C), $217.56(\mathrm{O}=\mathrm{C}) . \mathrm{MS}(\mathrm{EI}), \mathrm{m} / z$ (\%) 220.0 $\left(\mathrm{M}^{+}, 3\right), 133.8(100)$.

2,2-Dimethyl-5-(4-nitrophenyl)-dihydrofuran-3(2H)-one (2i). Yellow oil. ${ }^{1} \mathrm{H} \mathrm{NMR}\left(\mathrm{CDCl}_{3}\right) \delta$ $1.35\left(\mathrm{~s}, 3 \mathrm{H},-\mathrm{CH}_{3}\right), 1.43\left(\mathrm{~s}, 3 \mathrm{H},-\mathrm{CH}_{3}\right), 2.49(\mathrm{dd}, J=10.5 \mathrm{~Hz} ; J=18 \mathrm{~Hz}, 1 \mathrm{H}, \mathrm{H}-4 \mathrm{a}), 2.97$ (dd, $J=6$ $\mathrm{Hz} ; J=18 \mathrm{~Hz}, 1 \mathrm{H}, \mathrm{H}-4 \mathrm{~b}), 5.33$ (dd, $J=10.5 \mathrm{~Hz} ; J=6 \mathrm{~Hz}, 1 \mathrm{H},-\mathrm{CH}), 7.58-7.61$ (m, 2H, phenyl-H), 
8.24-8.27 (m, 2H, phenyl-H). ${ }^{13} \mathrm{C}$ NMR $\left(\mathrm{CDCl}_{3}\right) \delta 21.61\left(-\mathrm{CH}_{3}\right), 24.28\left(-\mathrm{CH}_{3}\right), 43.78(\mathrm{C}-4), 73.34$ (C-5), 81.79 (C-2), 123.96 (phenyl-C), 126.62 (phenyl-C), 147.65 (phenyl-C), 148.16 (phenyl-C), $215.58(\mathrm{O}=\mathrm{C})$. MS (EI), m/z (\%) $235.1\left(\mathrm{M}^{+}, 1\right), 149.0$ (100). HRMS Calcd. for $\mathrm{C}_{12} \mathrm{H}_{13} \mathrm{NO}_{2}$ 235.0845 , found 235.0851 .

2,2-Dimethyl-5-(naphthalen-2-yl)-dihydrofuran-3(2H)-one (2j). Yellow solid, mp 63-64 ${ }^{\circ} \mathrm{C}$. ${ }^{1} \mathrm{H}$ NMR $\left(\mathrm{CDCl}_{3}\right) \delta 1.38\left(\mathrm{~s}, 3 \mathrm{H},-\mathrm{CH}_{3}\right), 1.46\left(\mathrm{~s}, 3 \mathrm{H},-\mathrm{CH}_{3}\right), 2.66(\mathrm{dd}, J=10.5 \mathrm{~Hz} ; J=18.5 \mathrm{~Hz}, 1 \mathrm{H}$, $\mathrm{H}-4 \mathrm{a}), 2.95$ (dd, $J=6 \mathrm{~Hz} ; J=18 \mathrm{~Hz}, 1 \mathrm{H}, \mathrm{H}-4 \mathrm{~b}), 5.39$ (dd, $J=10.5 \mathrm{~Hz} ; J=6 \mathrm{~Hz}, 1 \mathrm{H},-\mathrm{CH})$, 7.47-7.52 (m, 3H, phenyl-H), 7.84-8.89 (m, 4H, phenyl-H). ${ }^{13} \mathrm{C}$ NMR $\left(\mathrm{CDCl}_{3}\right) \delta 21.73\left(-\mathrm{CH}_{3}\right)$, $24.44\left(-\mathrm{CH}_{3}\right), 44.11$ (C-4), 74.56 (C-5), 81.55 (C-2), 123.68 (naphthyl-C), 125.03 (naphthyl-C), 126.19 (naphthyl-C), 126.38 (naphthyl-C), 127.76 (naphthyl-C), 128.00 (naphthyl-C), 128.68 (naphthyl-C), 133.22 (naphthyl-C), 137.97 (naphthyl-C), 217.18 (O=C). MS (EI), m/z (\%) 240.0 $\left(\mathrm{M}^{+}, 5\right), 153.9$ (100). HRMS Calcd. for $\mathrm{C}_{16} \mathrm{H}_{16} \mathrm{O}_{2} 240.1150$, found 240.1163.

5-(Furan-2-yl)-2,2-dimethyl-dihydrofuran-3(2H)-one (2k). Yellow oil. ${ }^{1} \mathrm{H}$ NMR $\left(\mathrm{CDCl}_{3}\right) \delta$ $1.30\left(\mathrm{~s}, 3 \mathrm{H},-\mathrm{CH}_{3}\right), 1.33\left(\mathrm{~s}, 3 \mathrm{H},-\mathrm{CH}_{3}\right), 2.80(\mathrm{dd}, J=7 \mathrm{~Hz} ; J=18 \mathrm{~Hz}, 1 \mathrm{H}, \mathrm{H}-4 \mathrm{a}), 2.93(\mathrm{dd}, J=9 \mathrm{~Hz}$; $J=18 \mathrm{~Hz}, 1 \mathrm{H}, \mathrm{H}-4 \mathrm{~b}), 5.25$ (dd, $J=9 \mathrm{~Hz} ; J=7 \mathrm{~Hz}, 1 \mathrm{H},-\mathrm{CH}), 6.37-6.40$ (m, 2H, furyl-H), 7.44 (m, $1 \mathrm{H}$, furyl-H). ${ }^{13} \mathrm{C} \mathrm{NMR}\left(\mathrm{CDCl}_{3}\right) \delta 22.28\left(-\mathrm{CH}_{3}\right), 24.29\left(-\mathrm{CH}_{3}\right), 39.71(\mathrm{C}-4), 67.74(\mathrm{C}-5), 80.97$ (C-2), 108.50 (furyl-C), 110.39 (furyl-C), 143.10 (furyl-C), 152.37 (furyl-C), 216.35 (O=C). MS (EI), $m / z(\%) 180.1\left(\mathrm{M}^{+}, 5\right), 94.0$ (100). HRMS calcd. for $\mathrm{C}_{10} \mathrm{H}_{12} \mathrm{O}_{3}$ 180.0786, found 180.0795.

\section{Acknowledgements}

This work was financially supported by the National Natural Science Foundation of China (NSFC, 20876149), and the Natural Science Foundation of Zhejiang Province (Y406380).

\section{References}

1. Larhed, M.; Moberg, C.; Hallberg, A. Acc. Chem. Res. 2002, 35, 717.

2. Caddick, S.; Fitzmaurice, R. Tetrahedron 2009, 65, 3325.

3. Lidstrom, P.; Tierney. J.; Wathey, B.; Westman, J. Tetrahedron 2001, 45, 9225.

4. Zare, A.; Hasaninejad, A.; Beyzavi, M. H.; Zare, A. R. M.; Khalafi-Nezhad, A.; Asadi, F.; Baramaki, L.; Jomhori-Angali, S.; Ghaleh-Golabi, R. Synth. Commun. 2009, 39,139.

5. Khunt, R. C.; Akbari, J. D.; Manvar, A. T.; Tala, S. D.; Dhaduk, M. F.; Joshi, H. S.; Shah, A. Arkivoc 2008, (xi) 277.

6. Yadav, J. S.; Reddy, B. V. S.; Basak, A. K.; Narsaiah, A. V. J. Mol. Catal. A: Chemical 2007, $274,105$.

7. Jung, M. E. Stabilized Nucleophiles with Electron Deficient Alkenes and Alkynes. In Comprehensive Organic Synthesis; B. M. Trost, I. Fleming, Eds.; Pergamon Press: Oxford, 1991; Vol. 4, pp 1-67. 
8. Pelmutter, P. Conjugate Addition Reactions in Organic Synthesis; Pergamon Press: Oxford, 1992.

9. Nising, C. F.; Braese, S. Chem. Soc. Rev. 2008, 37, 1218.

10. Kisanga, P. B.; Ilankumaran, P.; Fetterly, B. M.; Verkade, J. G. J. Org. Chem. 2002, 67, 3555.

11. Stewart, I. C.; Bergman, R. G.; Toste, F. D. J. Am. Chem. Soc. 2003, 125, 8696.

12. Murtagh, J. E.; McCooey, S. H.; Connon, S. J. Chem. Commun. 2005, 227.

13. Farnworth, M. V.; Cross, M. J.; Louie, J. Tetrahedron Lett. 2004, 45, 7441.

14. Miller, K. J.; Kitagawa, T. T.; Abu-Omar, M. M. Organometallics 2001, 20, 4403.

15. Munro-Leighton, C.; Delp, S. A.; Blue, E. D.; Gunnoe, T. B. Organometallics 2007, 26, 1483.

16. Yang, L.; Xu, L. W.; Xia, C. G. Tetrahedron Lett. 2005, 46, 3279.

17. Wabnitz, T. C.; Spencer, J. B. Org. Lett. 2003, 5, 2141.

18. Bernal, P.; Tamariz, J. Tetrahedron Lett. 2006, 47, 2905.

19. Prabhakar, P.; Suryakiran, N.; Narasimhulu, M.; Venkateswarlu, Y. J. Mol. Catal. A: Chemical 2007, 274, 72.

20. Ramachary, D. B.; Mondal, R. Tetrahedron Lett. 2006, 47, 7689.

21. Kano, T.; Tanaka, Y.; Maruoka, K. Tetrahedron Lett. 2006, 47, 3039.

22. Karodia, N.; Liu, X. H.; Ludley, P.; Pletsas, D.; Stevenson, G. Tetrahedron 2006, 62, 11039.

23. Merschaert, A.; Delbeke, P.; Daloze, D.; Dive, G. Tetrahedron Lett. 2004, 45, 4697.

24. Biddle, M. M.; Lin, M.; Scheidt, K. A. J. Am. Chem. Soc. 2007, 129, 3830.

25. Dittmer, C.; Raabe, G..; Hintermann, L. Eur. J. Org. Chem. 2007, 5886.

26. Saito N.; Ryoda A.; Nakanishi W.; Kumamoto T.; Ishikawa T. Eur. J. Org. Chem. 2008, 2759.

27. Reiter M.; Turner H.; Gouverneur V. Chem. Eur. J. 2006, 12, 7190.

28. Wang, L.; Liu, X.; Dong, Z.; Fu, X.; Feng, X. Angew. Chem. Int. Ed. 2008, 47, 8670.

29. Brennan, C. M.; Hunt, I.; Jarvis, T. C.; Johnson, C. D.; McDonnell, P. D. Can. J. Chem. 1990, 68, 1780.

30. Baldwin, J. E.; Thomas, R. C.; Kruse, L. I.; Silberman, L. J. Org. Chem. 1977, 42, 3846.

31. Ellis, G. W. L.; Johnson, C. D.; Rogers, D. N. J. Am. Chem. Soc. 1983, 105, 5090.

32. Shen, Z. L.; Jiang, X. Z.; Mo, W. M.; Hu, B. X.; Sun, N. Green. Chem. 2005, 7, 97.

33. Ohnemuller, U. K.; Nising, C. F.; Nieger, M.; Brase, S. Eur. J. Org. Chem. 2006, 1535.

34. Ellis, G. W. L.; Johnson, C. D.; Rogers, D. N. J. Chem. Soc., Chem. Commun. 1982, 36.

35. Wabnitz, T. C.; Yu, J. Q.; Spencer, J. B. Chem. Eur. J. 2004, 10, 484.

36. Nazarov, I. N. Bull. Acad. Sci. URSS, Classe Sci. Chim. 1948, 107. Chem. Abstr. 1948, 42, 7737. 